\title{
Radioquimioterapia
}

\section{Avances en radio-quimioterapia en tumores cerebrales}

\author{
A. Ramos Aguerri, A. Hervás, R. Morera, A. Montero, S. Córdoba, J. Corona, I. Rodríguez, S. Sancho
}

\section{Introducción}

Los tumores cerebrales, tanto benignos como malignos constituyen una preocupación muy importante en todos aquellos profesionales implicados en el tratamiento de los mismos.

Así como en los tumores benignos, con la mejora en los últimos años tanto de las técnicas quirúrgicas como en el desarrollo de técnicas radioterápicas de alta precisión, (radiocirugía, radioterapia estereotáxica fraccionada, etc), se ha conseguido una mejora en el control de los mismos; en los tumores malignos de alto grado y aún a pesar del uso de todas estas técnicas modernas y su combinación con quimioterapia, los resultados obtenidos distan mucho de provocar satisfacción.

La incidencia oscila entre 5 y 70 casos por 100.000/habitantes/año dependiendo de la edad e incrementándose con la misma, existiendo dos picos en la presentación, uno en la edad infantil y otro entre 50-70 años con tendencia a aumentar. Estos tumores representan alrededor del $50 \%$ de todos los tumores cerebrales en el adulto siendo los más frecuentes y provocan alrededor del $2 \%$ de todas las muertes por cáncer.

El mal pronóstico de estos tumores se debe fundamentalmente a su alta malignidad pero también a unas particularidades propias de los mismos derivados del órgano donde asientan. El cerebro, órgano importantísimo desde el punto de vista vital y funcional, está ubicado dentro de una cavidad rígida que impide la expansión del tumor sin provocar graves perjuicios, ya que volúmenes tumorales superiores a $100 \mathrm{gr}$. son prácticamente incompatibles con la vida. Asimismo, al carecer de linfáticos y tener un menor flujo sanguíneo provoca una dificultad en el drenaje del edema peritumoral, contribuyendo esto al efecto de masa e incrementan-

Servicio de Oncología Radioterápica

Hospital Ramón y Cajal

Madrid do el problema de la hipoxia y la necrosis. La existencia de la barrera hematoencefálica dificulta la llegada de ciertos quimioterápicos, con el consiguiente perjuicio. En cuanto a radiosensibilidad, por las características intrínsecas, tanto de las células del tejido normal del sistema nervioso como de las células malignas que forman estos tumores, las dosis eficaces necesarias para el tratamiento curativo son muy similares a las dosis tóxicas.

En este artículo nos vamos a referir a aquellos tumores malignos de alto grado, que asientan en el sistema nervioso central como son los gliomas y en particular a los astrocitomas anaplásicos y glioblastomas multiformes. Estos tumores, a pesar de los avances llevados a cabo en la última década tanto en el diagnóstico como en el tratamiento siguen teniendo muy mal pronóstico.

Los factores pronósticos clásicos y en los que se basan prácticamente todos los estudios realizados y publicados en la literatura son: la edad, el tamaño tumoral, la histología, el estado general y el déficit neurológico en el momento del diagnóstico.

Tradicionalmente se ha considerado que la cirugía es el tratamiento de elección debiendo realizarse la extirpación máxima tumoral con la preservación de la función neurológi$\mathrm{ca}$, en la medida que sea posible. La supervivencia mediana de estos tumores tras cirugía es de unas 14 semanas en aquellos cuya intención terapéutica ha sido la curativa. Dadas las características de los mismos con tendencia a la infiltración tumoral de los tejidos próximos, la resección total de estos tumores conlleva una gran dificultad.

El tratamiento radioterápico postoperatorio ha contribuido a mejorar el control local y la supervivencia global, alcanzando una supervivencia mediana de 36 semanas. La quimioterapia asociada ha mejorado ligeramente esta supervivencia en este tipo de tumores.

De la revisión de los resultados publicados en la literatura resulta difícil sacar conclusiones, dada la variedad de factores pronósticos y diferentes terapéuticas utilizadas.

Como ya hemos dicho, la historia natural de estos tumores de alto grado, en general se caracteriza por su crecimiento local, siendo muy rara la diseminación a través de líquido cefalorraquídeo así como la metastatización fuera del sistema nervioso central. Por tanto, las recidivas, las cuales se presentan muy frecuentemente, la mayoría de las veces son locales. 


\section{A. Ramos Aguerri y cols.}

\section{Tratamiento radioterápico}

Se han publicado seis ensayos aleatorizados, comparando cirugía sóla frente a cirugía y radioterapia postoperatoria, objetivándose en cinco de ellos, que la radioterapia postoperatoria obtiene una beneficio significativo en la supervivencia de estos pacientes. En uno de ellos, en que este beneficio no se objetiva, un factor pronóstico como es el estado general está mal balanceado.

También ha existido controversia en cual debería ser el volumen que debería irradiarse en este tipo de tumor. En la etapa previa a la posibilidad de utilizar TAC y/o RMN para la delimitación adecuada de los volúmenes tumorales y de los órganos de riesgo, lo habitual era irradiar todo el volumen cerebral, a dosis de 40-45 Gy y reducción del mismo posteriormente, ajustándose al volumen tumoral y alcanzar dosis de 60-65 Gy.

A partir de 1989 en los estudios de Sapiro y Kita, no existían diferencias significativas en la supervivencia de los pacientes en los cuales la irradiación era mucho más limitada, realizándose exclusivamente el tratamiento a un volumen que comprendía el GTV con un margen de 2 a $4 \mathrm{~cm}$.

En cuanto a las dosis utilizadas para el control de estos tumores, no se ha demostrado diferencia significativa utilizando dosis superiores a 60 Gy con fraccionamiento clásico, siendo el control inferior cuando las dosis eran menores a esta. Así pues tanto en el estudio de Medical Research Council Brain Tumor Working Party como en el de RTOG/ECOG, ambos aleatorizados y con gran número de pacientes, 443 y 626 respectivamente, demuestran una mejoría tanto en la supervivencia mediana, como en la supervivencia global.

Asimismo, en un intento de mejorar el control de la enfermedad, se han utilizado distintos esquemas de fraccionamientos alterados, tanto hiperfraccionamiento, fraccionamientos acelerados, como radioterapia hipofraccionada, no habiéndose encontrado diferencias significativas respecto a radioterapia convencional.

Los tumores cerebrales en general, en el momento del diagnóstico, el $95 \%$ de los mismo son unifocales y más del $90 \%$ recidivan en el lugar del primario. Ante estas características se ha intentando mejorar el control local de esta enfermedad tratando de intensificar la dosis a ese nivel. Para ello se ha utilizado la braquiterapia como técnica de irradiación ya que sus características técnicas permiten administrar dosis elevadas en la zona tumoral con un decrecimiento muy rápido de la dosis conforme nos alejamos de la fuente de irradiación, permitiendo la preservación del tejido cerebral próximo.

En los estudios revisados se encuentra un escaso beneficio en la supervivencia mediana en aquellos pacientes en los que se ha utilizado braquiterapia, apreciándose un incremento de la misma en 2 meses.

Asimismo, se han utilizado terapias con partículas, neutrones, iones de helio, etc. tratando de mejorar la eficacia de los fotones debido al mayor efecto biológico y la mejor distribución de dosis en el volumen tumoral de estas partículas.

Hasta el momento actual no parece que el uso de esa tecnología, tan sofisticada, haya mejorado los resultados obtenidos con el uso terapéutico de los fotones.

Los importantes avances tecnológicos desarrollados y que se han incorporado al uso cotidiano de los tratamientos ra- dioterápicos como el uso de TAC y RMN en los nuevos planificadores, permiten realizar conformaciones tridimensionales más ajustadas al volumen a irradiar, disminuyendo el volumen de tejido normal que recibe una dosis de irradiación significativa. Todo esto permite aumentar las dosis a nivel tumoral y por tanto mejorar las posibilidades de incrementar el control local así como de disminuir la toxicidad derivada de esta preservación del tejido sano.

Una posible desventaja del uso de esta tecnología es que la definición de los volúmenes tumorales es más crítica y por lo tanto se debe ser muy cuidadoso en el diseño de los mismos ya que puede incrementarse la posibilidad de recidivas en el límite del volumen si estos márgenes no son los adecuados. Esta tecnología ha permitido alcanzar dosis a nivel tumoral de hasta 90 Gy con resultados discordantes respecto al control a nivel local, no habiéndose traducido en ningún caso en la mejora de la supervivencia.

Tambien se ha utilizado la radiocirugía, como técnica de alta precisión, administrándose una dosis única como sobreimpresión tras radioterapia convencional y en los estudios publicados en los que se mide supervivencia toxicidad, calidad de vida y estado mental, no existen diferencias significativas.

Es necesario conocer los resultados del estudio aletorizado de la RTOG/93-05, para saber si realmente la radiocirugía presenta una ventaja sobre el tratamiento convencional.

\section{Quimioterapia}

En los últimos años se han publicado numerosos trabajos en los que se objetiva una mejora en el control local y en la supervivencia con el uso de quimioterápicos combinados con radioterapia. Es sabido que la combinación de radioquimioterapia potencia la respuesta terapéutica en muchos tumores sólidos. Los mecanismos de acción de este tipo de tratamiento combinado ya fueron comentados por Steel y Peckan en 1979. La forma en que se han utilizado los distintos fármacos junto con la radioterapia ha sido tratando de potenciar la radioterapia, radiosensibilización, administrando dosis que en muchas ocasiones no son citotóxicas en sí mismas o bien utilizando quimioterápicos a dosis plenas, buscando además de una radiosensibilización, un efecto citotóxico y que en ocasiones no es posible realizarlo por incremento de los efectos tóxicos.

Entre el grupo de fármacos con los que se pretende una radiosensibilización se encuentran los sensibilizadores de células hipóxicas, pirimidinas halogenadas, perfluoroquininas, que transportan oxígeno a los tejidos, distintos quimioterápicos como el platino, fluoropirimidinas, taxanos, etc; inhibidores de la síntesis de poliamida intracelular que dificultan la reparación postirradiación. Los dos primeros grupos, sensibilizadores de células hipóxicas y pirimidinas, son los más estudiados.

En el grupo de los radiosensibilizadores de células hipóxicas están los mitroimidazoles, que aumentan la oxigenación disminuyendo la fracción de células hipóxicas tumorales y por tanto incrementado la radiosensibilización de dichas células.

Se han publicado dos metaanálisis utilizando metronidazol y misonidazol estudios de Overgaard y de Huncharek. En ninguno de ellos se han encontrado beneficios en el uso combinado de radiosensibilizadores e irradiación.

En cuando al uso de pirimidinas halogenadas, 5-bromo- 
deoxiuridina y 5-iododeoxiuridina, que son similares al precusor de la Timidina del ADN, en los que se ha sustituido un grupo metil por un halógeno, siendo las células que las incorporan más sensibles a las irradiaciones ionizantes y a la luz ultravioleta, no se han encontrado beneficios. El estudio realizado por la RTOG debió ser cerrado de forma prematura por que en el brazo experimental se obtuvo una peor supervivencia ( $68 \%$ versus $82 \%$ ).

Con el uso de la quimioterapia utilizada a dosis plenas y cuyos objetivos son incrementar la muerte celular y por tanto la supervivencia así como mejorar la calidad de vida de los pacientes, han sido publicados múltiples ensayos aleatorizados. En 1993, Fine en su metaanálisis, muestra la eficacia de la quimioterapia adyuvante mejorando la supervivencia de estos pacientes en un $8 \%$ a los dos años, aunque parece que esta mejora fundamentalmente se debe al mayor número de astrocitomas anaplásicos.

La mayoría de los ensayos aletorizados publicados se basan en la utilización de monoterapia sobre todo nitrosuras. En los estudios aleatorizados llevados a cabo por el Brain Tumor Study Group 69-01, 72-01, 75-01, 77-02, se comparan desde cuidados de soporte, CCNU como monoterapia, así como la utilización de radioterapia sola o combinada con $\mathrm{CCNU}$, administrándose los quimioterápicos tanto de forma concurrente como adyuvante. Con ellos se demuestra una mejora de la supervivencia mediana a 18 meses y que la supervivencia en el brazo experimental de radio-quimioterapia es de $19 \%$ versus $4 \%$ en el de radioterapia sola. Así mismo en el estudio de 75-01 se objetiva que los resultados con el uso de procarbacina son similares a los obtenidos con carmustina.

En cuanto a la utilización de monoterapia comparándola con la combinación de distintos quimioterápicos de forma concurrente a la radioterapia, en el estudio BTSG 80-01 con 571 pacientes, la combinación de carmustina, procarbacina y VM 26 no es superior a la utilización de carmustina sola. En otro estudio realizado por el Intergrupo de Estados Unidos en que se compara radioterapia con $\mathrm{CCNU} \pm$ cisplatino no se encuentran diferencias significativas añadiendo este último fármaco. Grossman en el estudio realizado en ECOG encuentra los mismos resultados.

En el estudio llevado a cabo por MRC Brain Tumor Working Party, en el que se compara radioterapia sola frente a radioterapia más procarbacina, CCNU y vincristina (PCV) no hay diferencias significativas en el control de este grupo de tumores.

Como ya se ha mencionado anteriormente en el metaanálisis llevado a cabo con 2.100 pacientes por Huncharek, en el que compara la monoterapia frente a la combinación, existe una pérdida de la supervivencia del $22 \%$ en el primer año en el brazo experimental de la combinación.

En los últimos años se han incorporado a la terapéutica de estos tumores, nuevos fármacos como el Gliadel ( Nitrosurea) y el Temodal (Temozolomida), aprobados actualmente en nuestro país, para el tratamiento de las recidivas. El primero, es una Nitrosurea presentanda en forma de implantes de polímeros y que tras la resección tumoral debe colocarse tapizando toda la cavidad quirúrgica. La liberación del quimioterápico es lenta y mantenida, constituyendo una nueva opción terapéutica. Aunque en España la indicación es en recidiva de astrocitomas anaplásicos y glioblastomas, sometidos a resección quirúrgica, el estudio más interesante es el publicado por $M$. Westphal, en el que realiza un ensayo ale- atorizado con 240 pacientes, a los cuales tras la resección inicial del tumor, llevan a cabo la implantación de Gliadel, en la cavidad quirúrgica, administrándose a continuación el tratamiento radioterápico estándar. Lo interesante en este estudio es que la supervivencia a largo plazo, a tres años, es superior de forma significativa (11\% versus $2 \%$ ) al grupo de pacientes que no habían recibido el tratamiento de carmustina (BCNU) en forma de polímero biodegradable.

En cuanto al segundo, temozolomida, es un agente alquilante oral, activo frente a gliomas, con propiedades radiosensibilizadoras. Actualmente está indicado en recidivas de gliomas, con respuestas que varían entre el 8 y $45 \%$ y con supervivencias medianas superiores a otros quimioterápicos utilizados en este tipo de tumor. La actividad radiosensibilizadora de la temozolomida viene dada por su mecanismo de acción, ya que es capaz de parar las células en fase G2 y $M$, que son las fases del ciclo celular más sensibles a la irradiación. Aprovechando este mecanismo sinérgico entre Temozolomida y radioterapia, Stupp utilizó dicho quimioterápico en concurrencia con la irradiación a dosis de 75 $\mathrm{mg} / \mathrm{m}^{2}$ / día durante seis semanas y una dosis de radioterapia de 60 Gy. Tras finalizar el tratamiento radioterápico, se continúa de forma adyuvante con Temozolomida a dosis de $200 \mathrm{mg} / \mathrm{m} 2$ x 5 y cada 28 días. Este estudio demostró que el tratamiento concurrente es factible y bien tolerado. La supervivencia obtenida a un año fue del $58 \%$ y de un $31 \%$ al segundo año con una supervivencia mediana de 16 meses.

Próximamente, se van a publicar los resultados de los ensayos aleatorizados, RTOG-9813 y EORTC/MCI de Canadá. En el primero se comparan temozolomida y carmustina y en el segundo la concurrencia de irradiación y Temodal frente a radioterapia sola.

\section{Nuevas dianas terapéuticas}

Las investigaciones realizadas en los últimos años nos ha llevado a un mejor conocimiento de las bases moleculares en el desarrollo del cáncer y el estudio de los tumores cerebrales no es una excepción.

En estos últimos se ha demostrado la sobreexpresión de diversos genes que codifican cascadas de señales que estimulan el crecimiento y la división celular. Asimismo se ha objetivado la pérdida de oncogenes supresores o de su función, como son p-53 o el gen del retinoblastoma. Así, basándose en estos conocimientos se han puesto en marcha numerosos estudios fase I y II, utilizando inhibidores de los receptores de tirosin-quinasa, farnesil-transferasa y topoisomerasa I. También se está estudiando la utilización de oligonucleótidos antisentido, con la finalidad de restaurar la función de diversos genes mutados. Además se ha abierto un campo de investigación, con numerosos estudios fase I y II, utilizando terapias con agentes biológicos, entre ellos el Interferon. La inmunoterapia en sus diversas formas adoptiva, vacuna de células dendríticas, diversas terapias génicas, inhibidores de la angiogénesis y MMP y Talidomida están en fases de plena investigación en el tratamiento de estos tumores.

Lo que es evidente es que se ha abierto un nuevo y amplio campo en la investigación del diagnóstico y tratamiento de los tumores malignos del sistema nervioso central basándose en el mejor conocimiento de las bases moleculares del cáncer, presentando un esperanzador futuro. 


\section{A. Ramos Aguerri y cols.}

\section{Bibliografía}

Long DM, Gordon T, Bowman H, et al. Outcome and cost of craniotomy performed to treat tumors in regional academic referral centers. Neurosurgery 2003; 52:1056.

Berger MS. Malignant astrocytomas: Surgical aspects. Semin Oncol 1994; $21: 172$.

Frankel SA, German WJ. Glioblastoma multiforme. Review of 219 cases with regard to natural history, pathology, diagnostic methods, and treatment. J Neurosurg 1958; 15:489.

Coffey RJ, Lunsford LD, Taylor FH. Survival after stereotactic biopsy of malignant gliomas. Neurosurgery 1988; 22:465.

Duncan CG, Goodman GB, Ludgate CM, et al. The treatment of adult supratentorial high grade astrocytomas. J Neurooncol 1992; 13:63.

Kreth FW, Warnke PC, Scheremet R, Ostertag CB. Surgical resection and radiation therapy versus biopsy and radiation therapy in the treatment of glioblastoma multiforme. J Neurosurg 1993; 78:762.

Simpson JR, Horton J, Scott C, et al. Influence of location and Andersen AP. Postoperative irradiation of glioblastomas. Results in a randomized series. Acta Radiol Oncol Radiat Phys Biol 1978, 17:475.

Walker MD, Alexander EJ, Hunt WE, et al. Evaluation of BCNU and/or radiotherapy in the treatment of anaplastic gliomas: $A$ cooperative clinical trial. J Neurosurg 1978; 49:333.

Walker MD, Strike TA, Sheline GE. An analysis of dose-effect relationship in the radiotherapy of malignant gliomas. Int J Radiat Oncol Biol Phys 1979; 5:1725.

Chang $\mathrm{CH}$, Horton J, Schoenfeld D, et al. Comparison of postoperative radiotherapy and combined postoperative radiotherapy and chemotherapy in the multidisciplinary management of malignant gliomas. A joint Radiation Therapy Oncology Group and Eastern Cooperative Oncology Group study. Cancer 1983, 52:997.

Nelson DF, Diener-West M, Horton J, et al. Combined modality approach to treatment of malignant gliomas-re-evaluation of RTOG 7401/ECOG 1374 with long-term follow-up: a joint study of the Radiation Therapy Oncology Group and the Eastern Cooperative Oncology Group. NCl Monogr $1988: 279$.

Shapiro WR. Therapy of adult malignant brain tumors: What have clinical trials taught us? Semin Oncol 1986; 13:38.

Hochberg FH, Pruitt A. Assumptions in the radiotherapy of glioblastoma. Neurology 1980; 30:907.

Wallner KE, Galicich JH, Krol G, et al. Patterns of failure following treatment for glioblastoma multiforme and anaplastic astrocytoma. Int J Radiat Oncol Biol Phys 1989; 16:1405.

Choucair AK, Levin VA, Gutin PH, et al. Development of multiple lesions during radiation therapy and chemotherapy in patients with gliomas. J Neurosurg 1986; 65:654.

Liang BC, Thornton AFJ, Sandler HM, et al. Malignant astrocytomas: Focal tumor recurrence after focal external beam radiation therapy. J Neurosurg 1991; 75:559.

TenHaken RK, Thornton AF Jr, Sandler HM, et al. A quantitative as sessment of the addition of MRI to CT-based, 3-D treatment planning of brain tumors. Radiother Oncol 1992; 25:121.

Thornton AFJ, Hegarty TJ, TenHaken RK, et al. Three-dimensional treatment planning of astrocytomas: A dosimetric study of cerebral irradiation. Int J Radiat Oncol Biol Phys 1991; 20:1309

Glatstein E, Lichter AS, Fraass BA, et al. The imaging revolution and radiation oncology: Use of $\mathrm{CT}$, ultrasound, and NMR for localization, treatment planning and treatment delivery. Int J Radiat Oncol Biol Phys 1985; 11:299.

Nakagawa K, Aoki Y, Fujimaki T, et al. High-dose conformal radiotherapy influenced the pattern of failure but did not improve survival in glioblastoma multiforme. Int J Radiat Oncol Biol Phys 1998; 40:1141.

Chan JL, Lee SW, Fraass BA, et al. Survival and failure patterns of high-grade gliomas after three-dimensional conformal radiotherapy. J Clin Oncol 2002; 20:1635.

Scharfen CO, Sneed PK, Wara WM, et al. High activity iodine-125 interstitial implant for gliomas. Int J Radiat Oncol Biol Phys 1992; 24:583.

Nehls DG, Shetter AG, Rossman KJ. Interstitial irradiation of malignant tumors. BNIQ 1985; 1:34.

Wilson $\mathrm{CB}$, Larson $\mathrm{DA}$, Gutin $\mathrm{PH}$. Radiosurgery: A new application? J Clin Oncol 1992; 10:1373.

Selker RG, Shapiro WR, Burge P, et al. The Brain Tumor Cooperative Group NIH Trial 87-01: a randomized comparison of surgery, external radiotherapy, and carmustine versus surgery, interstitial radiotherapy boost, external radiation therapy, and carmustine. Neurosurgery 2002; 51:343.

Laperriere NJ, Leung PM, McKenzie S, et al. Randomized study of brachytherapy in the initial management of patients with malignant astrocytoma. Int J Radiat Oncol Biol Phys 1998; 41:1005.

Bernstein $M$, Laperriere $N$, Leung $P$, et al. Interstitial brachytherapy for malignant brain tumors: Preliminary results. Neurosurgery 1990; 26:371

Cohen L, Awschalom M. Fast neutron radiation therapy. Annu Rev Biophys Bioeng 1982; 11:359.

Cohen L, Hendrickso FR, Kurup PD, et al. Clinical evaluation of neutron beam therapy. Current results and prospects, 1983. Cancer $1985 ; 55: 10$.

Griffin TW, Davis R, Laramore $G$, et al. Fast neutron radiation therapy for glioblastoma multiforme. Am J Clin Oncol 1983; 6:661.

Laramore GE. Neutron radiotherapy for high grade gliomas: The search for the elusive therapeutic window. Int J Radiat Oncol Biol Phys 1990; 19:493.

Castro JR, Saunders WM, Austin-Seymour MM, et al. A phase I-II trial of heavy charged particle irradiation of malignant glioma of the brain: A Northern California Oncology Group study. Int J Radiat Oncol Biol Phys 1985; 11:1795.

Castro JR, Phillips TL, Prados $M$, et al. Neon heavy charged particle radiotherapy of glioblastoma of the brain. Int J Radiat Oncol Biol Phys 1997; 38:257.

Laramore GE, Spence AM. Boron neutron capture therapy (BNCT) for high-grade gliomas of the brain: a cautionary note. Int J Radiat Oncol Biol Phys 1996; 36:241.

Dorn RVI. Boron neutron capture therapy (BNCT). A radiation oncology perspective. Int J Radiat Oncol Biol Phys 1994; 28:1189.

Loeffler JS, Alexander El, Kooy HM, et al. Radiosurgery for brain metastases. In: PPO updates: Cancer. Principles and practice of oncology, Devita, VT, Hellman, S, Rosenberg, SA, JB Lippincott, Philadelphia, 1991.

Leksell L. The stereotactic method and radiosurgery of the brain. Acta Chir Scand 1951; 102:316.

Shrieve DC, Alexander E 3rd, Black PM, et al. Treatment of patients with primary glioblastoma multiforme with standard postoperative radiotherapy and radiosurgical boost: prognostic factors and long-term outcome. J Neurosurg 1999; 90:72. 
Prisco FE, Weltman E, de Hanriot RM, Brandt RA. Radiosurgical boost for primary high-grade gliomas. J Neurooncol 2002; 57:151

Nwokedi EC, Di Biase SJ, Jabbour S, et al. Gamma knife stereotactic radiosurgery for patients with glioblastoma multiforme. Neurosurgery 2002; 50:41.

Curran WJ, Scott CB, Weinstein AS, et al. Survival comparison of radiosurgery-eligible and - ineligible malignant glioma patients treated with hyperfractionated radiation therapy and carmustine: A report of Radiation Therapy Oncology Group 83-02. J Clin Oncol 1993; 11:857.

Deutsch $M$, Green SB, Strike TA, et al. Results of a randomized trial comparing $\mathrm{BCNU}$ plus radiotherapy, streptozotocin plus radiotherapy, $\mathrm{BCNU}$ plus hyperfractionated radiotherapy, and $\mathrm{BCNU}$ following misonidazole plus radiotherapy in the postoperative treatment of malignant glioma. Int J Radiat Oncol Biol Phys 1989; 16:1389.

Nelson DF, Diener-West M, Weinstein AS, et al. A randomized comparison of misonidazole sensitized radiotherapy plus $B C N U$ and radiotherapy plus BCNU for treatment of malignant glioma after surgery: final report of an RTOG study. Int J Radiat Oncol Biol Phys 1986; 12:1793.

Huncharek M. Meta-analytic re-evaluation of misonidazole in the treatment of high grade astrocytoma. Anticancer Res 1998; 18:1935.

Prados MD, Scott C, Sandler H, et al. A phase 3 randomized study of radiotherapy plus procarbazine, $C C N U$, and vincristine (PCV) with or without BUdR for the treatment of anaplastic astrocytoma: a preliminary report of RTOG 9404. Int J Radiat Oncol Biol Phys 1999; 45:1109. 